\title{
Flow stress of biomorphous metal-matrix composites
}

\author{
Baohua $\mathrm{Ji}^{\mathrm{a}}$, Huajian $\mathrm{Gao}^{\mathrm{a}, *}$, Tzuchiang Wang ${ }^{\mathrm{b}}$ \\ a Max Planck Institute for Metals Research, Heisenbergstrasse 3, Stuttgart 70569, Germany \\ ${ }^{\mathrm{b}}$ LNM, Institute of Mechanics, Chinese Academy of Science, Beijing 100080, PR China
}

Received 18 May 2004; received in revised form 19 July 2004

\begin{abstract}
For metal-matrix composites (MMCs), interfacial debonding between the ductile matrix and the reinforcing hard inclusions is an important failure mode. A fundamental approach to improving the properties of MMCs is to optimize their microstructure to achieve maximum strength and toughness. Here, we investigate the flow stress of a MMC with a nanoscale microstructure similar to that of bone. Such a 'biomorphous' MMC would be made of staggered hard and slender nanoparticles embedded in a ductile matrix. We show that the large aspect ratio and the nanometer size of inclusions in the biomorphous MMC lead to significantly improved properties with increased tolerance of interfacial damage. In this case, the partially debonded inclusions continue to carry mechanical load transferred via longitudinal shearing of the matrix material between neighboring inclusions. The larger the inclusion aspect ratio, the larger is the flow stress and work hardening rate for the composite. Increasing the volume concentration of inclusion also makes the biomorphous MMC more tolerant of interfacial damage.

(C) 2004 Elsevier B.V. All rights reserved.
\end{abstract}

Keywords: Metal-matrix composite; Interfacial debonding; Unit cell model; Plasticity; Biomorphous materials; Bio-inspired materials

\section{Introduction}

Metal-matrix composites (MMCs) with high stiffness and high yield strength have attracted various applications in automobile, power plant and aircraft industries. Typically, an MMC is made of a ductile metallic matrix reinforced by a distribution of hard and brittle inclusions. A fundamental understanding of the relationship between the microstructure and the macroscopic behaviors can help optimize the flow stress and fracture energy of these materials. Previous studies [1-7] have shown that the mechanical properties of MMCs depend on the geometry, volume fraction and distribution of reinforcing inclusions. Fig. 1a shows the typical microstructure of MMCs. The relatively rigid (fiber-like or flake-like) ceramic inclusions can cause significant increase in elastic modulus, flow stress, and work hardening of the composite materials [1-7].

The addition of ceramic particles in MMCs can also have ramifications concerning plastic flow and fracture. Notably,

\footnotetext{
* Corresponding author. Tel.: +49 711 6893510; fax: +49 7116893512. E-mail address: hjgao@mf.mpg.de (H. Gao).
}

the mismatch in elastic/plastic properties and thermal expansion between the inclusions and matrix, lead to inhomogeneous plastic yielding and consequent void nucleation in matrix during loading [8,9]. These mismatches can induce large stresses within the inclusions or at the matrix-inclusion interfaces [10,11]. A number of failure modes in MMCs have been reported in the past, including matrix/particle debonding [12-16], particle cracking [11,17-24] and ductile failure in the matrix $[25,26]$. The elastic modulus, flow stress and ductility of MMCs are found to drop significantly as soon as such failures occur [27-29]. In particular, interface decohesion has been identified as one of the most frequently observed damage modes of MMCs, especially at high temperatures when the strength of inclusions is high compared to that of the interface between matrix and inclusion, as in aircraft engines. Zhao and Weng [30] and Ju and Lee [31] modeled the influence of interfacial debonding on the elastic-plastic behavior of particle reinforced composites. In their analysis, interfacial debonding along the long side of the particle is found to cause significant degradation to the composite properties.

Materials sensitive to localized flaws or damage are prone to catastrophic failure due to random nucleation events. It 

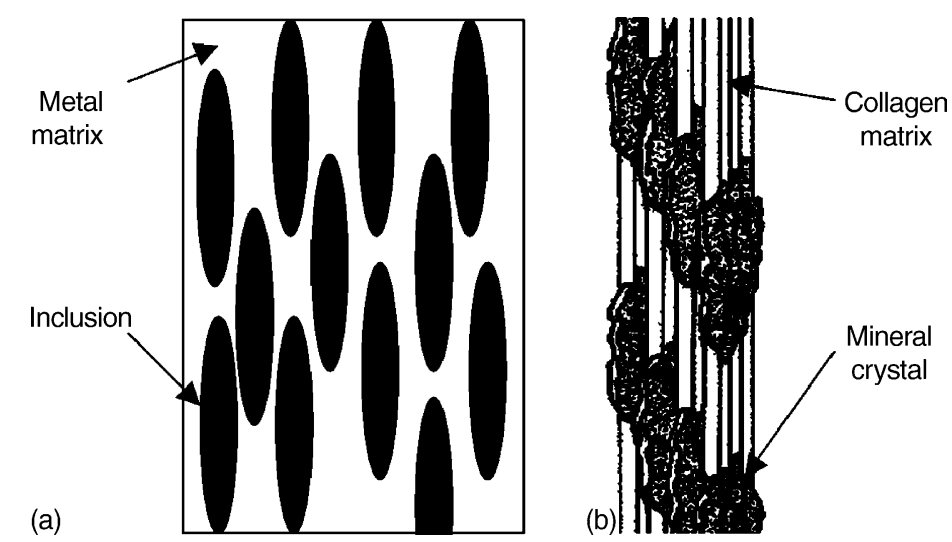

Fig. 1. Schematic illustrations of (a) the microstructure of a biomorphous metal matrix composite and (b) the nanostructure of bone; the latter consists of nanometer sized mineral crystals staggered in the collagen matrix.

is therefore important to search for ideas to increase the reliability of materials [32]. Here, we consider a nanostructured MMC, mimicking the nanoscale design of bone-like biological materials [33-38] and made of hard and slender inclusions embedded in a soft protein matrix (Fig. 1b). Recent experimental [35,39-41] and theoretical studies [42-45] have shown that the nanostructure of bone-like materials represents a convergent design of evolution and plays a crucial role in their macroscopic properties. Partial debonding along the mineral-protein interface does not lead to significant drop in stiffness and strength of bone, but rather serves as a feedback for initiation of biological remodeling [42-48]. Gao et al. $[42,43]$ pointed out that the nanometer-sized mineral inclusions can maintain their theoretical strength in the presence of crack-like flaws, and that the theoretical strength of inclusion is necessary to allow the protein matrix to dissipate large amount of fracture energy so as to achieve a high toughness for the composite.

This paper is aimed to investigate the flow stress of a biomorphous MMC with partial interfacial debonding. We define a biomorphous MMC as a ductile metallic matrix reinforced by hard and slender nanoparticles in a geometrical alignment similar to that in bone. A unit cell model is adopted in which the inclusion is treated as a hard elastic particle surrounded by an elastic-plastic matrix. The interface between the inclusion and matrix perpendicular to applied loading in the longitudinal direction is assumed to have debonded due to local tensile stress. The effects of debonding on the flow stress of the composite are calculated for various aspect ratios and volume concentrations of the inclusions. The influence of the aspect ratio of the inclusions on the work hardening rate of the composite is estimated by a tension-shear chain model (TSC) originally developed for the analysis of nanoscale properties of bone.

\section{Method of analysis}

We adopt a standard finite element scheme to evaluate the flow stress of a biomorphous MMC. The reader is referred to Llorca and Gonzalez [4] for details of the FEM scheme. Consider a two-dimensional representative volume and a unit cell of MMC as shown in Fig. 2. The unit cell takes into account the staggered alignment of inclusions in the matrix.

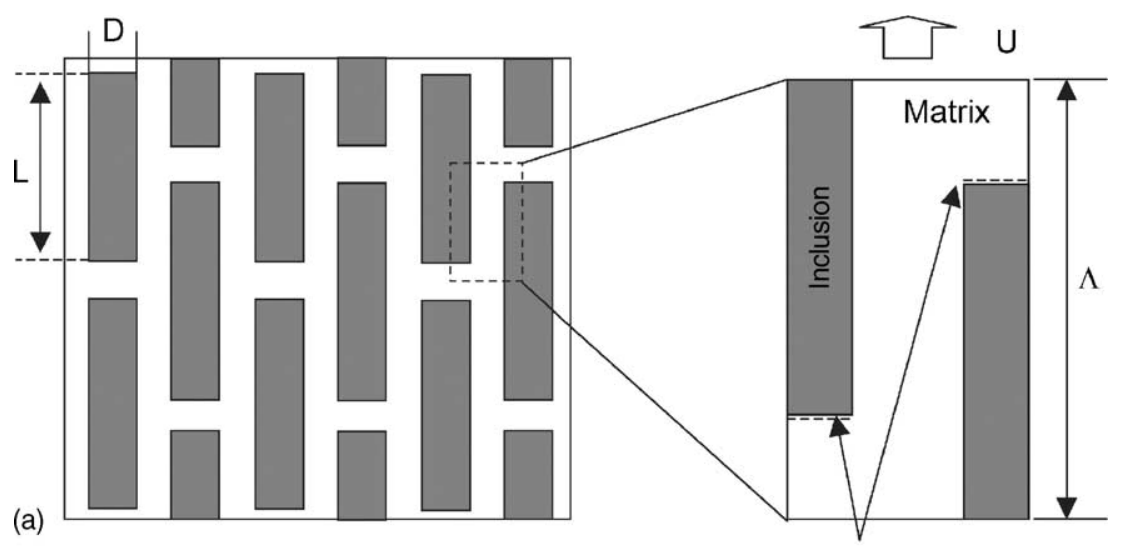

(b) Debonding

Fig. 2. (a) The representative volume element and (b) a unit cell model of biomorphous MMCs. Debonding at the long end of the inclusion is assumed to have occurred due to local tensile stress. 
The volume concentration of inclusion is $\Phi$. Debonding is assumed to have occurred along the inclusion-matrix interface perpendicular to the loading direction. The inclusions are assumed to be purely elastic and the matrix is modeled as an elastic-plastic solid following the $\mathbf{J}_{2}$ flow theory of plasticity. The flow stress of the matrix is represented by a power law

$\sigma_{\mathrm{M}}=\sigma_{0}\left(\frac{\varepsilon_{\mathrm{M}}^{\mathrm{p}}}{\varepsilon_{0}}\right)^{n}$,

where $\varepsilon_{\mathrm{M}}^{\mathrm{p}}, \sigma_{0}$ and $\varepsilon_{0}$ denote the plastic strain, reference stress, and reference strain, respectively, and $n$ is the strain hardening exponent of the matrix. A displacement $U$ is applied on the top side of the unit cell, as shown in Fig. 2. The composite strain in the direction of loading is calculated by

$E=\ln \left(\frac{U}{\Lambda}\right)$

where $\Lambda$ is the length of the unit cell. The inclusion has an aspect ratio $\rho=L / D$ where $L$ is the length and $D$ the thickness of the inclusion. The composite stress in the direction of loading is obtained by an average over the unit cell,

$\Sigma=\frac{1}{V} \int \sigma \mathrm{d} V$

The composite plastic strain can be calculated as

$E^{\mathrm{p}}=E-\frac{\Sigma}{Y}$,

where $Y$ is the effective modulus of the composite, which depends on the Young's moduli of the inclusion and the matrix, as well as the volume concentration of inclusion $\Phi$. In practice, the composite modulus $Y$ can be calculated based on Eqs. (2) and (3) by applying an infinitesimal deformation on the cell.

If the relation between plastic strain and stress of the matrix has the power-law form of Eq. (1), the corresponding relation of the composite can also be expressed as a power law based on the unit cell approach [4-7],

$\Sigma=Q \sigma_{0}\left(\frac{E^{\mathrm{p}}}{\varepsilon_{0}}\right)^{n}$

where $Q$ is called the strengthening factor which can be determined by [5-7],

$Q=\frac{\Sigma\left(E^{\mathrm{p}}\right)}{\sigma_{\mathrm{M}}\left(E^{\mathrm{p}}\right)}$

Eq. (5) has been confirmed by the finite element analysis of Llorca and Gonzalez [4]. In general, the value of $Q$ is determined by the plastic behavior of matrix as well as the geometry and volume concentration of inclusions.

\section{Flow stress of biomorphous MMC}

Consider a biomorphous MMC with interfacial debonding perpendicular to the loading direction. Due to the large aspect ratio of inclusion, the matrix-inclusion interface parallel to the loading direction is subjected to relatively small shear stress and will be assumed to remain intact during plastic deformation of the composite. The interface perpendicular to the loading direction is subjected to relatively large tension, where debonding will be assumed to test the damage tolerance and reliability of the MMC.

To obtain some insight into the effect of interfacial debonding, we first make a comparison between the plastic behavior of the pure matrix material and a debonded MMC at $\Phi=0.15, n=0.1$. The results (Fig. 3) indicate that, for equiaxed inclusions $(\rho=1)$, the flow stress of the composite is drastically decreased by the interfacial debonding, to a level even lower than that of the matrix. However, for large aspect ratios $(>5)$, the inclusions are able to retain their strengthening effect despite the debonding. The larger the aspect ratio, the stronger the strengthening effect. The ratio between the strengthening factor of a debonded composite and that of the corresponding intact composite, $Q / Q^{*}$, is plotted as a function of the aspect ratio in Fig. 4 for two different volume concentrations. These results show that large aspect ratios allow the debonded composite to retain a flow stress at a level close to that of an intact composite despite debonding. In addition, the biomorphous composites with large aspect ratios become more insensitive to interfacial debonding at larger volume concentration of inclusions.

The mechanism by which slender inclusions minimize the damage of interfacial deconding is that large aspect ratio reduces the average stress in the matrix and decreases the relative proportion of the loss of load capacity due to debonding. The inclusions take the main load in the composite structure. The force transfer between the adjacent inclusions is achieved via longitudinal shear deformation of the matrix. In contrast, the interface perpendicular to the loading direction plays a

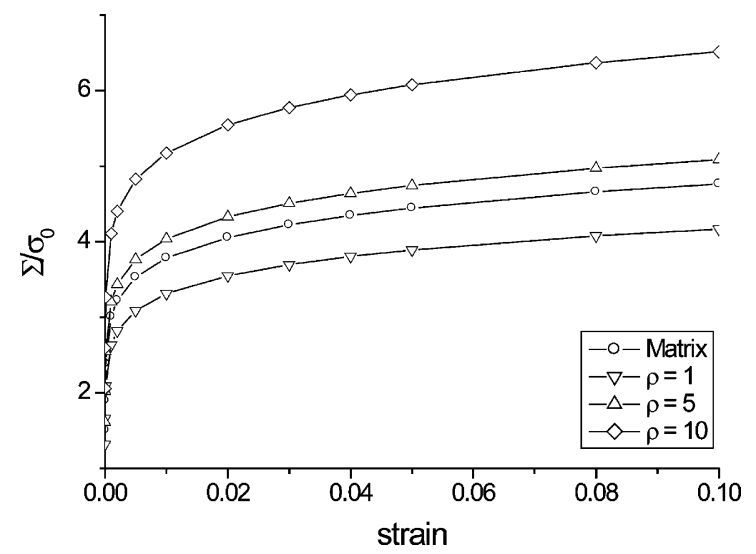

Fig. 3. The relation between stress and plastic strain of a biomorphous MMC with partial interfacial debonding in comparison with that of matrix at different aspect ratios of the inclusion. 


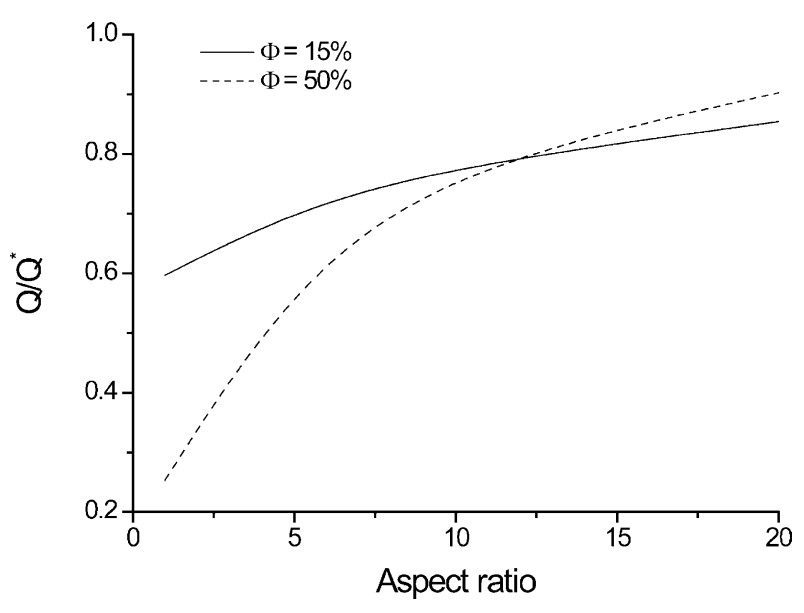

Fig. 4. The influence of the aspect ratio of the inclusion on the strengthening factor $Q$ at two different inclusion volume concentrations in the biomorphous MMC with partial debonding. The data is normalized by the strengthening factor $Q^{*}$ of the intact MMC.

relatively insignificant role in load transfer. Fig. 5 plots the stress level in the inclusion as a function of the aspect ratio at two different volume concentrations. The result shows that the stress level in the inclusion increases with the aspect ratio (i.e. more strengthening). For sufficiently large aspect ratios, the stress level in the damaged composite is nearly the same as that in the corresponding intact composite (see Figs. 4 and 5).

\section{Work hardening rate of biomorphous MMC}

The biomorphous MMC has similar nanostructure morphology as bone. Fig. 6a illustrates the primary route of load transfer in the nanostructure of bone, which can be represented by a TSC model. In this tension-shear chain system, mineral inclusions carry tensile load and the soft matrix transfers load between hard inclusions via shear. Following the analysis of Gao et al. $[42,43]$, the maximum stress $\sigma_{\mathrm{I}}$ in the

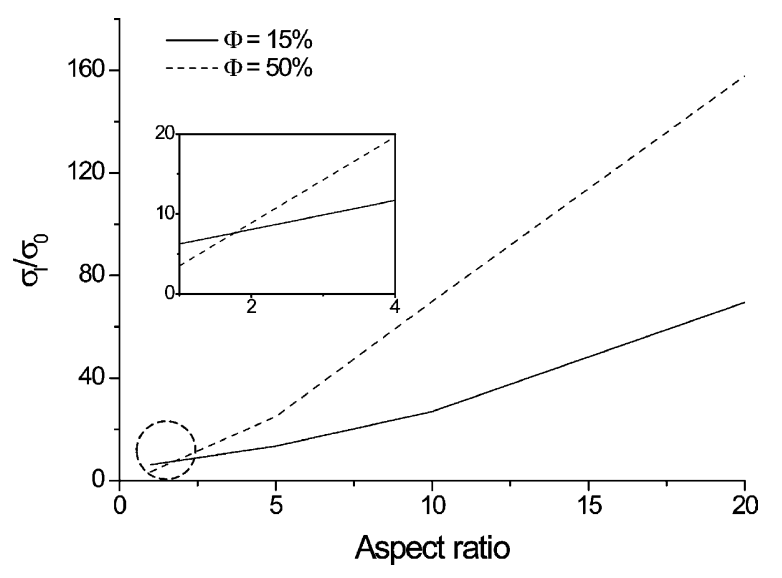

Fig. 5. The stress $\sigma_{\mathrm{I}}$ of the inclusion vs. its aspect ratio under an external load. The plot is normalized by the initial yield stress $\sigma_{0}$ of the matrix.

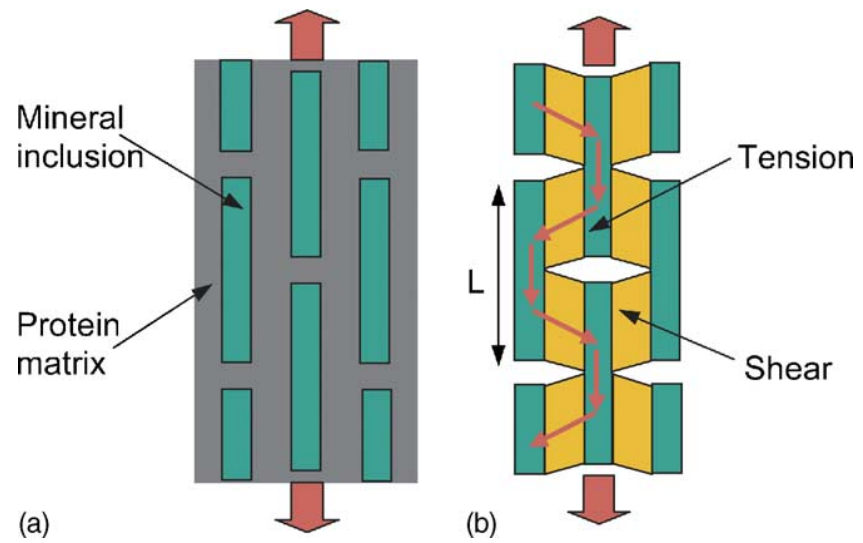

Fig. 6. Schematic illustration of the generic nanostructure of bone-like materials. Hard mineral crystals align in a soft protein matrix to sustain tensile loads. The soft matrix serves as glue layers to transfer forces between neighboring inclusions via shear. The large aspect ratio allows the soft layer to transfer a large force with relatively small shear stress.

inclusion can be related to the average shear stress $\tau_{M}$ in the matrix in an incremental form as

$\Delta \sigma_{\mathrm{I}}=\rho \Delta \tau_{\mathrm{M}}$

The large aspect ratio thus reduces the matrix stress. The incremental composite stress can be written as

$\Delta \Sigma=\frac{1}{2} \Phi \Delta \sigma_{\mathrm{I}}=\frac{1}{2} \rho \Phi \Delta \tau_{\mathrm{M}}$,

where $\Phi$ is the volume concentration of mineral. The incremental composite strain obeys the following kinematic relation,

$\Delta E=\frac{\Delta \delta_{\mathrm{I}}+2 \Delta \gamma_{\mathrm{M}} D(1-\Phi) / \Phi}{L}$,

where $\Delta \delta_{\mathrm{I}}$ and $\Delta \gamma_{\mathrm{M}}$ are the incremental elongation of the inclusion and incremental shear strain of the matrix, which can be expressed as

$\Delta \delta_{\mathrm{I}}=\frac{\Delta \sigma_{\mathrm{I}} L}{2 Y_{\mathrm{I}}}, \quad \Delta \gamma_{\mathrm{M}}=\frac{\Delta \tau_{\mathrm{M}}}{\mu_{\mathrm{M}}}$,

where $\mu_{\mathrm{M}}$ is the shear modulus of the matrix, and $Y_{\mathrm{I}}$ the Young's modulus of the inclusion. The composite modulus can thus be derived from Eqs. (7)-(10) as

$\frac{1}{Y}=\frac{4(1-\Phi)}{\mu_{\mathrm{M}} \Phi^{2} \rho^{2}}+\frac{1}{\Phi Y_{\mathrm{I}}}$.

The derivation steps of these equations are identical to those in $[42,43]$ except an incremental form of equations is used here. Eq. (11) indicates that a large aspect ratio $\rho$ allows the matrix to transfer a large tensile load on inclusions with relatively small shear stress.

For MMCs, plastic deformation in the metal matrix is confined by the inclusions. Strong size effects due to plastic strain gradients in the metal matrix may be present [49-51]. Here we focus on the effect of inclusion aspect ratio and temporarily neglect the strain gradient effect. With these assumptions, 
the relation between flow stress and plastic strain in the matrix can be written in an incremental form as

$\Delta \sigma_{\mathrm{M}}^{\mathrm{p}}=h \Delta \varepsilon_{\mathrm{M}}^{\mathrm{p}}$,

where according to Eq. (1)

$h=\frac{n \sigma_{0}}{\varepsilon_{0}}\left(\frac{\varepsilon_{\mathrm{M}}^{\mathrm{p}}}{\varepsilon_{0}}\right)^{n-1}$,

If we assume $n \leq 0.1$ (engineering metals usually fall in this category), then $h \leq 0.1 Y_{\mathrm{M}}$ under sufficiently large plastic deformation (e.g. $\varepsilon_{\mathrm{M}}^{\mathrm{p}}>\varepsilon_{0}$ ), $Y_{\mathrm{M}}$ being the Young's modulus of the matrix. The modulus ratio between inclusion and matrix in MMCs can be as large as 10 , i.e. $Y_{\mathrm{M}} \approx 0.1 Y_{\mathrm{I}}$, and thus the tangent modulus $h$ can be approximately two-orders of magnitude smaller than the Young's modulus of the inclusion, i.e., $h \leq 0.01 Y_{\mathrm{I}}$. As the inclusions take an incremental tensile load with modulus $Y_{\mathrm{I}}$, the matrix undergoes plastic deformation with a much smaller tangent modulus $h$. For an incremental plastic strain $\Delta \gamma_{\mathrm{M}}^{\mathrm{p}}$ in the biomorphous MMC, the incremental shear stress $\Delta \tau_{\mathrm{M}}^{\mathrm{p}}$ can be written as

$\Delta \tau_{\mathrm{M}}^{\mathrm{p}}=h^{\prime} \Delta \gamma_{\mathrm{M}}^{\mathrm{p}}$,

where $h^{\prime}=2 h / 3$. Replacing the second equation of (10) by (14), the tangent work hardening rate of the composite is obtained analogous to Eq. (11) as

$\frac{1}{H}=\frac{4(1-\Phi)}{h^{\prime} \Phi^{2} \rho^{2}}+\frac{1}{\Phi Y_{\mathrm{I}}}$.

An implicit assumption behind Eq. (15) is that the matrix material between two neighboring inclusions can be treated as a shear lag under uniform shear deformation. Fig. 7 shows that the prediction of Eq. (15) is in good agreement with the FEM calculation of the composite work hardening rate $H$ versus the inclusion aspect ratio (at plastic strain $E^{\mathrm{p}}=0.1$ ).

Inclusions with large aspect ratios help enhance the work hardening of the composite. On the other hand, the stress level within the inclusion increases with the aspect ratio (Fig. 5),

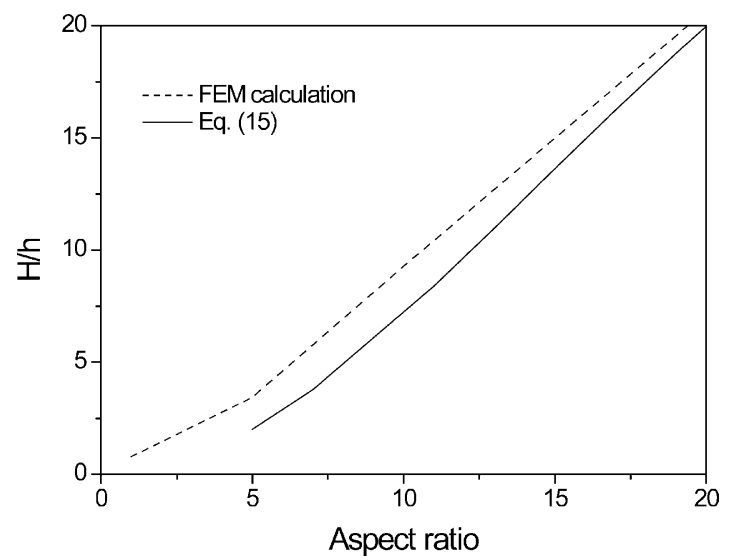

Fig. 7. Prediction of Eq. (15) on the tangent work hardening rate $H$ of a biomorphous $\mathrm{MMC}$ vs. the inclusion aspect ratio at plastic strain $E^{\mathrm{p}}=0.1$. The result is compared to the calculation of a full FEM analysis. and too large an aspect ratio will cause brittle fracture of the inclusion. An optimum value of $\rho$ should exist depending on the fracture strength and the volume concentration of inclusions. As has been discussed in Gao et al. [42] and Gao and Ji [52], the fracture strength of a brittle inclusion containing a crack-like flaw depends on the size of the inclusion as,

$\frac{\sigma_{\mathrm{I}}^{\mathrm{f}}}{\sigma_{\mathrm{th}}}=\left\{\begin{array}{c}\sqrt{\frac{D^{*}}{D}}, \quad D \geq D^{*} \\ 1, \quad D<D^{*}\end{array}\right.$,

where $\sigma_{\text {th }}$ is the theoretical strength of the inclusion and $D^{*}$ can be estimated as

$D^{*} \approx \alpha^{2} \frac{\gamma Y_{\mathrm{m}}}{\sigma_{\mathrm{th}}^{2}}$.

Taking a typical flaw as a thumbnail crack spanning half the thickness of the inclusion, $D^{*}$ is estimated to be around $30 \mathrm{~nm}$ under rough estimates of $\gamma=1 \mathrm{~J} / \mathrm{m}^{2}, Y_{\mathrm{m}}=100 \mathrm{GPa}$, and $\sigma_{\text {th }}$ $=Y_{\mathrm{m}} / 30$. If the fracture strength of Eq. (16) is taken into account, we obtain an optimum aspect ratio as a function of inclusion size and volume concentration, as shown in Fig. 8. Taking $D^{*}$ to be $30 \mathrm{~nm}$, it is found that when the inclusion size falls into the submicron range, the optimum aspect ratio is in the range of 10-40, roughly the same as in shell and bone.

Therefore, a close analogy exists between bone-like materials and biomorphous MMCs. The stiffness and strength of biocomposites require the mineral crystals to have a large aspect ratio and nanometer size. In comparison, biomorphous MMCs achieve large flow stress and work hardening rate also via large aspect ratio and nanometer size of the reinforcing inclusions. As the inclusion size varies from a few tens to a few hundreds of nanometers, there exists an optimum aspect ratio for the reinforcing inclusions in a biomorphous MMC in the range of 10-40. Partial interfacial debonding has little effect on the biomorphous MMC. Such damage actually plays a beneficial role to animals. Studies on bone $[47,48]$ showed that damage in the cement lines of cortical bone provides a message for damage sensors to initiate the biological remodeling process. This concept may be used in the future

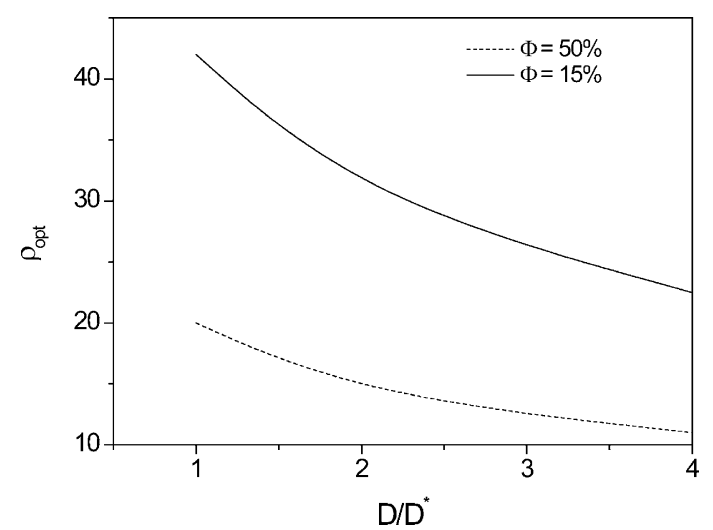

Fig. 8. The optimum aspect ratio as a function of the size and volume concentration of the inclusion in a biomorphous MMC. 
to develop smart materials in which interfacial debonding may be used to sense localized damage and subsequently triggers an alert system or self-repair of the structure. In both biomorphous MMCs and bone, the nanometer size of the slender inclusions is necessary to prevent brittle fracture in these inclusions. Consequently, the large aspect ratio of the inclusions leads to large flow stress and large work hardening rate in the MMC.

We have so far focused on material properties in the direction parallel to the aligned inclusions. Such model MMC is expected to be very anisotropic. The properties in the transverse directions are usually not significantly improved by the platelets. To develop an effective MMC in response to complex 3D loading, one may need to develop hierarchical laminate strategies similar to those of biological composites. In nature, mineralized collagen fibrils in bone which have been reinforced along the fiber direction often form complex 3D fibrous network. How nature arranges fiber layout to achieve optimized properties on the macroscopic scale is still not fully understood. Much future study is still needed on investigating hierarchical anisotropic microstructures to achieve interesting macroscopic materials properties.

Biological systems are usually constructed as laminates at nanoscale. One interesting question is whether this is a realistic possibility for MMCs in the future. Construction of hierarchical laminate structures is of course a severe challenge for the present technology. However, manufacturing techniques such as rapid prototyping are already opening up the possibility to construct sophisticated multilayered materials. Through rapid deposition and evaporation methods, it may become possible in the future to design laminate structures from nanoscale and up. The present paper is only a preliminary theoretical effort in this direction.

\section{Summary}

Flaw tolerance is a key to robust structure design and the robustness is the key to survival. The present study of biomorphous MMCs has been motivated by the study of bone-like biological materials which have the nanostructure of hard and slender mineral nanoparticles staggered in a protein matrix. A biomorphous MMC has been defined as a ductile metallic matrix reinforced by hard and slender nanoparticles in a geometrical alignment similar to that in bone. We have investigated the influence of aspect ratio, size and volume concentration of inclusions on the flow stress and work hardening rate of a biomorphous MMC with partial interfacial debonding. The microstructure of the composite was represented by a unit cell through which the flow stress of MMCs was calculated. The analysis indicated a strong analogy between biomorphous MMCs and biological materials in several aspects. The larger the aspect ratio of the inclusion, the bigger the strengthening effects. The composite becomes insensitive to interfacial debonding when the aspect ratio is sufficiently large. There exists an optimum aspect ratio which depends on the inclusion size and volume concentration. Like in biological materials, small size (nanometer) and large aspect ratio (10-40) of the inclusion are the key factors contributing to a robust microstructure of MMCs. We also found that increasing the volume concentration of inclusion makes the biomorphous MMC more tolerant of interfacial damage at large aspect ratio.

\section{Acknowledgments}

We acknowledge generous support from the Max-Planck Society, the Overseas Young Investigator Award by the National Science Foundation of China and the Chang Jiang Scholar program by the Ministry of Education of China. TW is supported by the National Natural Science Foundation of China (No. 19704100), National Science Foundation of Chinese Academy of Sciences (Project KJ951-1-20).

\section{References}

[1] T. Christman, A. Needleman, S. Suresh, Acta Metall. 37 (1989) 3029.

[2] G. Bao, J.W. Hutchinson, R.W. McMeeking, Acta Metall. Mater. 39 (1991) 1871.

[3] A.G. Evans, J.W. Hutchinson, R.M. McMeeking, Scripta Metall. Mater. 25 (1991) 3.

[4] J. Llorca, C. Gonzalez, J. Mech. Phys. Solids 46 (1998) 1.

[5] H.T. Zhu, H.M. Zbib, Int. J. Plast. 11 (1995) 471.

[6] B. Ji, T.C. Wang, Acta Mech. Sin. (English Series) 15 (1999) 344.

[7] B. Ji, T.C. Wang, Int. J. Plast. 19 (2003) 565.

[8] S.F. Corbin, D.S. Wilkinson, Acta Metall. Mater. 42 (1994) 1319.

[9] D.B. Zahl, R.M. McMeeking, Acta Metall. Mater. 39 (1991) 1117.

[10] B. Wilner, J. Mech. Phys. Solids 36 (1988) 141.

[11] J.R. Brockenbrough, F.W. Zok, Acta Metall. Mater. 43 (1995) 11.

[12] A.F. Whitehouse, T.W. Clyne, Acta Metall. Mater. 41 (1993) 1701.

[13] C.A. Lewis, W.M. Stobbs, P.J. Withers, Mater. Sci. Eng. A 171 (1993) 1

[14] S.R. Nutt, J.M. Duva, Scripta Metall. 20 (1986) 1055.

[15] A. Needleman, J. Appl. Mech. 54 (1987) 525.

[16] S.R. Nutt, A. Needleman, Scripta Metall. 21 (1987) 705.

[17] W.H.J. Hunt, J.R. Brockenbrough, P.E. Magnusen, Scripta Metall. Mater. 25 (1991) 15.

[18] D.J. Lloyd, Acta Metall. Mater. 39 (1991) 59.

[19] P.M. Singh, J.J. Lewandowski, Metall. Trans. A 24 (1993) 2531.

[20] J. Llorca, A. Martin, J. Ruiz, M. Elices, Metall. Trans. A 24 (1993) 1575.

[21] Y. Brechet, J.D. Embury, S. Tao, L. Luo, Acta Metall. Mater. 39 (1991) 1781.

[22] C.-W. Nan, D.R. Clarke, Acta. Mater. 44 (1996) 3801.

[23] M.T. Kiser, F.W. Zok, D.S. Wilkinson, Acta. Mater. 44 (1996) 3465.

[24] M. Finot, Y.-L. Shen, A. Needleman, S. Suresh, Metall. Mater. Trans. A 25 (1994) 2403.

[25] J. Lewandowski, C. Liu, W.H. Hunt, Mater. Sci. Eng., A 107 (1989) 241.

[26] M. Geni, M. Kikuchi, Acta Mater. 46 (1998) 3125.

[27] G. Bao, Acta Metall. Mater. 40 (1992) 2547.

[28] M.S. Hu, Scripta Metall. Mater. 25 (1991) 695.

[29] J. Llorca, P. Poza, Mater. Sci. Eng. A 185 (1994) 25.

[30] Y.H. Zhao, G.J. Weng, Int. J. Plast. 12 (1996) 781.

[31] J.W. Ju, H.K. Lee, Int. J. Solids Struct. 38 (2001) 6307.

[32] A.G. Evans, Acta. Mater. 45 (1997) 23. 
[33] J.D. Currey, Proc. R. Soc. London, Ser. B 196 (1977) 443.

[34] A.P. Jackson, J.F.V. Vincent, R.M. Turner, Proc. R. Soc. London, Ser. B 234 (1988) 415.

[35] S. Kamat, X. Su, R. Ballarini, A.H. Heuer, Nature 405 (2000) 1036.

[36] J.Y. Rho, L. Kuhn-Spearing, P. Zioupos, Med. Eng. Phys. 20 (1998) 92.

[37] S. Weiner, H.D. Wagner, Ann. Rev. Mater. Sci. 28 (1998) 271.

[38] I. Jaeger, P. Fratzl, Biophys. J. 79 (2000) 1737.

[39] P. Fratzl, H.F. Jakob, S. Rinnerthaler, P. Roschger, K. Klaushofer, J. Appl. Cryst. 30 (1997) 765

[40] W.J. Landis, Bone 16 (1995) 533.

[41] W.J. Landis, K.J. Hodgens, J. Struct. Biol. 117 (1996) 24.

[42] H. Gao, B. Ji, I.L. Jäger, E. Arzt, P. Fratzl, Proc. Natl. Acad. Sci. U.S.A. 100 (2003) 5597.
[43] H. Gao, B. Ji, M.J. Buehler, H. Yao, Mech. Chem. Biosystems 1 (2004) 37.

[44] B. Ji, H. Gao, Mater. Sci. Eng. A 366 (2004) 96.

[45] B. Ji, H. Gao, J. Mech. Phys. Solids 52 (2004) 1963.

[46] R.Z. Wang, Z. Suo, A.G. Evans, N. Yao, I.A. Aksay, J. Mater. Res. 16 (2001) 2485.

[47] H.M. Frost, in: H.D. Uhtoff (Ed.), Current Concepts of Bone Fragility, Springer, Berlin, 1985, pp. 123-148.

[48] T.L. Norman, Z. Wang, Bone 20 (1997) 375.

[49] W.D. Nix, H. Gao, J. Mech. Phys. Solids 46 (1998) 411.

[50] H. Gao, Y. Huang, W.D. Nix, J.W. Hutchinson, J. Mech. Phys. Solids 47 (1999) 1239.

[51] Z. Xue, Y. Huang, M. Li, Acta Mater. 50 (2002) 149.

[52] H. Gao, B. Ji, Eng. Fract. Mech. 70 (2003) 1777. 